\title{
Analysis of Tongue Color-Associated Features among Patients with PCR-Confirmed COVID-19 Infection in Ukraine
}

\author{
Liudmyla Horzov1@, Myroslav Goncharuk-Khomyn²@, Natalia Hema-Bahyna ${ }^{3}(\mathbb{D}$, Anastasia \\ Yurzhenko ${ }^{4}$ (D), Volodymyr Melnyk ${ }^{5}$ (D)
}

\begin{abstract}
'Department of Therapeutic Dentistry, Uzhhorod National University, Uzhhorod, Ukraine.
${ }^{2}$ Department of Prosthetic Dentistry, Uzhhorod National University, Uzhhorod, Ukraine.

${ }^{3}$ Department of Clinical Disciplines, Uzhhorod National University, Uzhhorod, Ukraine.

${ }^{4}$ Department of Dentistry of Postgraduate Education, Uzhhorod National University, Uzhhorod, Ukraine.

${ }^{5}$ Department of Pediatric Dentistry, Uzhhorod National University, Uzhhorod, Ukraine.
\end{abstract}

Correspondence: Myroslav Goncharuk-Khomyn, Uzhhorod National University, Universitetska 16/a st., Uzhhorod, 88000, Ukraine. E-mail: myroslav.goncharuk-khomyn@uzhnu.edu.ua

Academic Editor: Alessandro Leite Cavalcanti

Received: 19 January 2021 / Review: 24 February 2021 / Accepted: 05 March 2021

How to cite: Horzov L, Goncharuk-Khomyn M, Hema-Bahyna N, Yurzhenko A, Melnyk V. Analysis of tongue colorassociated features among patients with PCR-confirmed Covid-19 infection in Ukraine. Pesqui Bras Odontopediatria Clín Integr. 2021; $21: \mathrm{e} 0011$. https://doi.org/10.1590/pboci.2021.109

\begin{abstract}
Objective: To evaluate and systematize tongue color-related manifestations among patients with PCRconfirmed COVID-19 infection. Material and Methods: This retrospective study included analysis of tongue images obtained from patients with PCR-confirmed COVID-19 infection. Evaluation of coronavirus disease severity (mild, moderate, severe, critical) was provided, considering clinical symptomatology and results of laboratorial and instrumental diagnostic methods. Each picture was analyzed considering the parameters of color of the tongue and color of the tongue plaque by two dental specialists. CochranArmitage test for trend was used to evaluate associations between the tongue color and tongue plaque color, and coronavirus disease severity. Results: The most prevalent tongue colors were pale pink, red and dark red (burgundy color). A total of $64.29 \%$ of patients with mild disease demonstrated pale pink color of the tongue. Patients with moderate coronavirus disease were characterized with the adverse trend: $62.35 \%$ of them presented with red-colored tongue, while in $37.64 \%$ of cases, the tongue was pale pink. Severe COVID-19 patients, almost in $90 \%$ of the cases, had either red or burgundy color of the tongue. Conclusion: SARS-COV-2 infection is not manifested by tongue-targeted or tongue-specific signs and features; however, coronavirus disease itself provokes changes within the tongue color and tongue plaque color similar to those registered during other internal pathologies.
\end{abstract}

Keywords: Coronavirus Infections; Severe Acute Respiratory Syndrome; Tongue. 


\section{Introduction}

State of health could be indirectly evaluated by the analysis of the tongue color and the color of plaque accumulated on it before and during the clinical course of different internal diseases [1-5]. A clean tongue without plaque is a sign of a healthy person, while some of the somatopathologies are characterized with adjacent tongue manifestations, which either could be pathognomonic or included within the set of general symptoms [6].

Tongue-related features could be used as valuable diagnostic signs for health evaluation purposes under the machine learning processing approach, which includes the implementation of multi-modal algorithms to relate tongue changes (as image biomarkers) with other symptoms and pathological conditions (including coronavirus disease) [7-10]. Nevertheless, tongue features seem to be mostly related to the syndrome rather than the disease itself [11]. Previously it was found that lung diseases, such as tuberculosis, amyotrophic lateral sclerosis, a chronic obstructive pulmonary disease characterized with corresponding tongue manifestations [12-15].

Globally at the date of 13th of January 2021, there have been more than 90 million confirmed cases of COVID-19 reported to WHO, including 1,954,336 deaths [16]. In Ukraine, more than 1 million confirmed cases of COVID-19 with 20,214 deaths have been reported to the above-mentioned date [16]. Considering the COVID-19 pandemic situation, ongoing study of the manifestations related to this pathology within the oral cavity, particularly on the tongue, remains as an important practical and scientific objective [17-20]. The most common oral symptoms among persons who have suffered from a coronavirus infection include punctate hemorrhages in the form of red bumps, candidiasis, ulcers and various other lesions of the tongue and mucous membranes [17-19].

Chemosensory dysfunctions regarding smell and taste are considered one of the most prevalent screening signs among patients with influenza-like symptomatology, who could potentially be diagnosed as COVID-positive [21-23]. The nature of the COVID-related taste disorder remains under further investigation since this sign could be conceivably associated with developing hyposalivation, changes in saliva composition and neurological alterations [21,22].

Several studies have already investigated tongue-related features as the symptoms of COVID-19 and found statistically significant differences between COVID-19 patients and healthy people [24,25]. But there are only a small number of publications that address the topic of COVID-associated changes of the tongue related to the coronavirus disease severity. Previously it was found that the level of greasy coating and tenderness of the tongue are the most diagnostically sensitive signs for COVID-19 severity differentiation [26]. Pang et al. [26] concluded that studies regarding the quantification of tongue manifestations during coronavirus disease should be provided with the purpose to improve diagnostics, differentiation, and treatment of COVID-19 during the present pandemic era.

This study aimed to evaluate and systematize tongue color-related manifestations among patients with PCR-confirmed COVID-19 infection considering disease severity.

\section{Material and Methods}

\section{Study Design and Data Collection}

The design of the study was retrospective and included analysis of tongue images obtained from patients with PCR-confirmed COVID-19 infection. Analyzed patients' cohort was formed out of persons hospitalized in the reorganized COVID-Department of Uzhhorod Regional Clinical Hospital with COVID- 
associated symptomatology and further identified having SARS-COV2 by PCR method using oropharyngeal and nasal swabs samples.

Tongue pictures were obtained by the infectious disease specialist, who was directly providing treatment for COVID-19 patients in the corresponding department using smartphone devices without considering some specific technical characteristics of photodocumentation due to the relevant sanitary restrictions limited possibilities to obtain high-quality photos. Each image was taken with no magnification effect and no filters used. As a result, tongue photos were received with respect to all implemented restrictions and sanitary requirements while not compromising any affiliated risk of infection for medical personal or patients.

Obtained tongue pictures were coded and anonymized with the additional description provided just for the severity of coronavirus disease associated with each photo at the day of its' reception, age, and gender of the patient. Evaluation of coronavirus disease severity (mild, moderate, severe, critical) was provided by professional infectionists due to the World Health Organization recommendations [27], considering clinical symptomatology of the patients and results of additional laboratorial and instrumental diagnostic methods.

Binary informational blocks in the amount of 167 pairs consisted of tongue photo in *.jpeg format and text info with a description of verified COVID-19 severity, patient's age and gender were sent to the Department of Therapeutic Dentistry (Uzhhorod National University) via restricted access to Google Drive folder. Each picture was analyzed considering the parameters of color of the tongue and color of the tongue plaque by two dental specialists (experts) independently. Experts were assigned from the personal of the Department of Therapeutic Dentistry and Department of Prosthetic Dentistry (Uzhhorod National University) (chosen experts were previously calibrated by taking participation in various epidemiological and clinical studies).

With the purpose to exclude the effect of the interpretation error, which could occur during expert evaluation and comparison of study object (tongue image) with some addressable classification' referent, dental specialists who provided analysis of the tongue pictures were asked to describe them considering the color of the tongue or the color of tongue plaque without referring to any classification or categorization system by one or two color-associated adjective words.

Out of 167 tongue images, 32 were excluded from the study sample due to the presence of blurring effect, critical contrasting, cropping of some tongue part on the picture and moving-associated artefacts.

\section{Data Analysis}

Statistical argumentation of obtained differences considering correspondences between the tongue color and tongue plaque color to COVID-19 severity within groups of patients with mild, moderate and severe stages of the coronavirus disease was based on inter-groups' prevalence parameters comparison [28-30]. Cochran-Armitage test for trend was used to evaluate associations between the tongue color and tongue plaque color and coronavirus disease severity [31,32]. Such an approach was modified from Pang et al. [26], at which authors used the Chi-square test for trend analysis. Registered outcome values were validated as statistically significant if $\mathrm{p}<0.05$. Reproducibility of two dental specialists who provided analysis of tongue images considering the evaluation of the tongue color and tongue plaque color was calculated by the Cohen's kappa, parameters of which reached $0.83 \pm 0.06$ and $0.80 \pm 0.09$ respectively, while inter-rater percentage agreement was $86.7 \%$ and $83.4 \%$ respectively [32,33]. Final categorization of obtained tongue images, received description and numerical values were provided due to the following targeted parameters: tongue color, tongue plaque color, patient's age group (due to the 10 years period distribution), patient's gender (male/female). 
Statistical data processing was held within Microsoft Excel software (Microsoft Office 2019, Microsoft Corp., USA) with the additional use of add-ins, such as Analyse-it (Analyse-it Software Ltd., Leeds, UK) and XLSTAT (Addinsoft Inc., New York, N.Y, USA).

Ethical Aspects

This study was previously affirmed by the Ethical Committee of Faculty of Dentistry at Uzhhorod National University 2020 (Protocol No. 1/10092020). All the patients signed modified informed consent form included in their general medical documentation, which contained the section about the possibility of using their medical data with the research and investigation purposes for the improvement of medical care quality during COVID-19 era while providing full guarantee and security of personal issues (including obligatory anonymization) and in the correspondence of established ethical principles.

\section{Results}

Among 135 patients, who's tongue images were analyzed, 64 were males $(47.41 \%)$ and 71 were females (52.59\%). The average age was of the patients was $48.7 \pm 13.2$ years varying in the range from 18 to 82 years old. Distribution of patients regarding age criteria presented in Table 1.

Table 1. Distribution of patients with PCR-confirmed cases of COVID19 due to the age criteria.

\begin{tabular}{ccc}
\hline Age Group (Years) & N & \% \\
\hline$<20$ & 8 & 5.88 \\
$21-30$ & 15 & 11.03 \\
$31-40$ & 17 & 12.50 \\
$41-50$ & 28 & 20.50 \\
$51-60$ & 23 & 16.91 \\
$61-70$ & 17 & 12.50 \\
$71-80$ & 14 & 10.29 \\
$>80$ & 13 & 9.56 \\
\hline
\end{tabular}

"The distribution of patients regarding coronavirus disease severity was as follows: 14 (10.37\%) patients with mild coronavirus disease severity; $85(62.96 \%)$ patients with moderate coronavirus disease severity; 36 (26.67\%) patients with severe coronavirus disease; and no patients with critical severity of pathology were observed in this study.

The most prevalent tongue colors were pale pink, red and dark red (burgundy color). A percentual of $64.29 \%$ of patients $(n=9)$ with mild disease demonstrated pale pink color of the tongue, while the rest, $35.71 \%$ $(\mathrm{n}=5)$, had a red-colored tongue. Patients with moderate coronavirus disease were characterized with adverse trend: $62.35 \%$ of them $(n=53)$ presented with red-colored tongue, while in $37.64 \%$ cases $(n=32)$, the tongue was pale pink. Severe COVID-19 patients almost in $90 \%$ of the cases $(n=32)$ had either red or burgundy color of the tongue, while the rest of the patients $(11.11 \% ; n=4)$ in this group had pale pink tongue color (Figure 1).

A total of $91.11 \%$ of patients $(n=123)$ were characterized by the presence of tongue plaque. Patients with no tongue plaque were distributed among those having mild $(n=9)$ and moderate $(n=3)$ coronavirus disease, while $100 \%$ of patients with severe pathology demonstrated the presence of tongue plaque.

The most common colors of the tongue plaque were white, gray, yellow. Among patients with mild disease, no plaque was found in $64.28 \%$ of patients $(n=9)$, while the other $35.71 \%$ of patients $(n=5)$ had a white plaque. Patients with moderate disease severity were mostly characterized with white (15.29\%; n=13), yellow $(25.88 \% ; n=22)$ and gray $(55.29 \% ; n=47)$ plaque. A total of $3.52 \%$ of patients $(n=3)$ with moderate disease 
demonstrated no plaque present on the tongue. Patients presented with severe coronavirus disease had a white plaque in 27 cases $(75.0 \%)$ and yellow plaque in 9 cases $(25.0 \%)$. Visually amount of white plaque in severe patients' cases was thicker than in mild or moderate diseases cases (Table 2 and Figure 2 ).

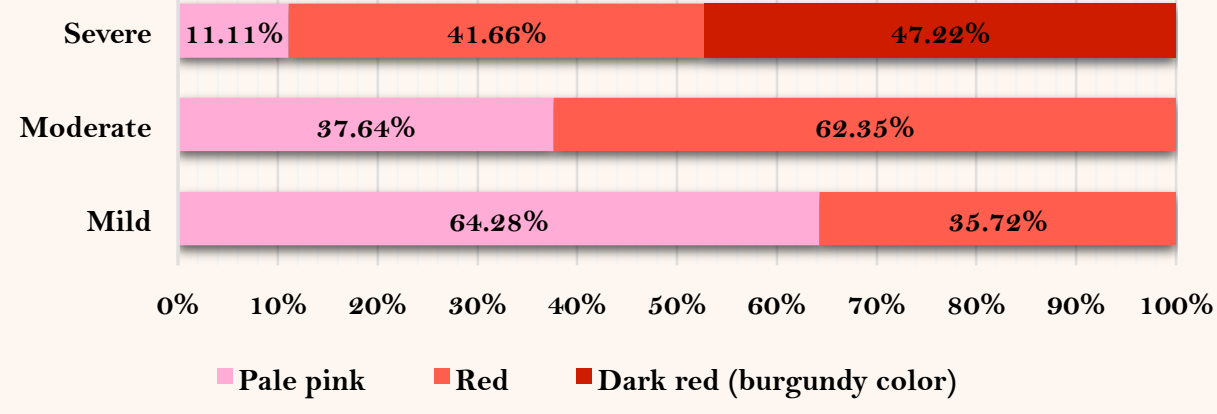

Figure 1. Distribution of tongue colors among COVID-19 patients with different disease severity.

Table 2. Tongue features related to coronavirus disease severity.

\begin{tabular}{|c|c|c|c|c|}
\hline \multirow[t]{2}{*}{ Tongue Features } & \multirow[t]{2}{*}{ Categories } & \multicolumn{3}{|c|}{ Coronavirus Disease Severity } \\
\hline & & $\begin{array}{l}\text { Mild } \\
\mathrm{N}(\%) \\
\end{array}$ & $\begin{array}{c}\text { Moderate } \\
\mathrm{N}(\%)\end{array}$ & $\begin{array}{l}\text { Severe } \\
\mathrm{N}(\%)\end{array}$ \\
\hline \multirow[t]{3}{*}{ Tongue Color } & Pale Pink & $9(64.28)$ & $32(37.64)$ & $4(11.11)$ \\
\hline & Red & $5(35.71)$ & $52(62.35)$ & $15(41.66)$ \\
\hline & Dark Red (Burgundy Color) & - & - & $17(47.22)$ \\
\hline \multirow[t]{4}{*}{ Tongue Plaque Color } & White & $5(35.71)$ & $13(15.29)$ & $27(75.0)$ \\
\hline & Grey & - & $47(55.29)$ & - \\
\hline & Yellow & - & $22(25.88)$ & $9(25.0)$ \\
\hline & No Plaque Present & $9(64.28)$ & $3(3.52)$ & - \\
\hline
\end{tabular}

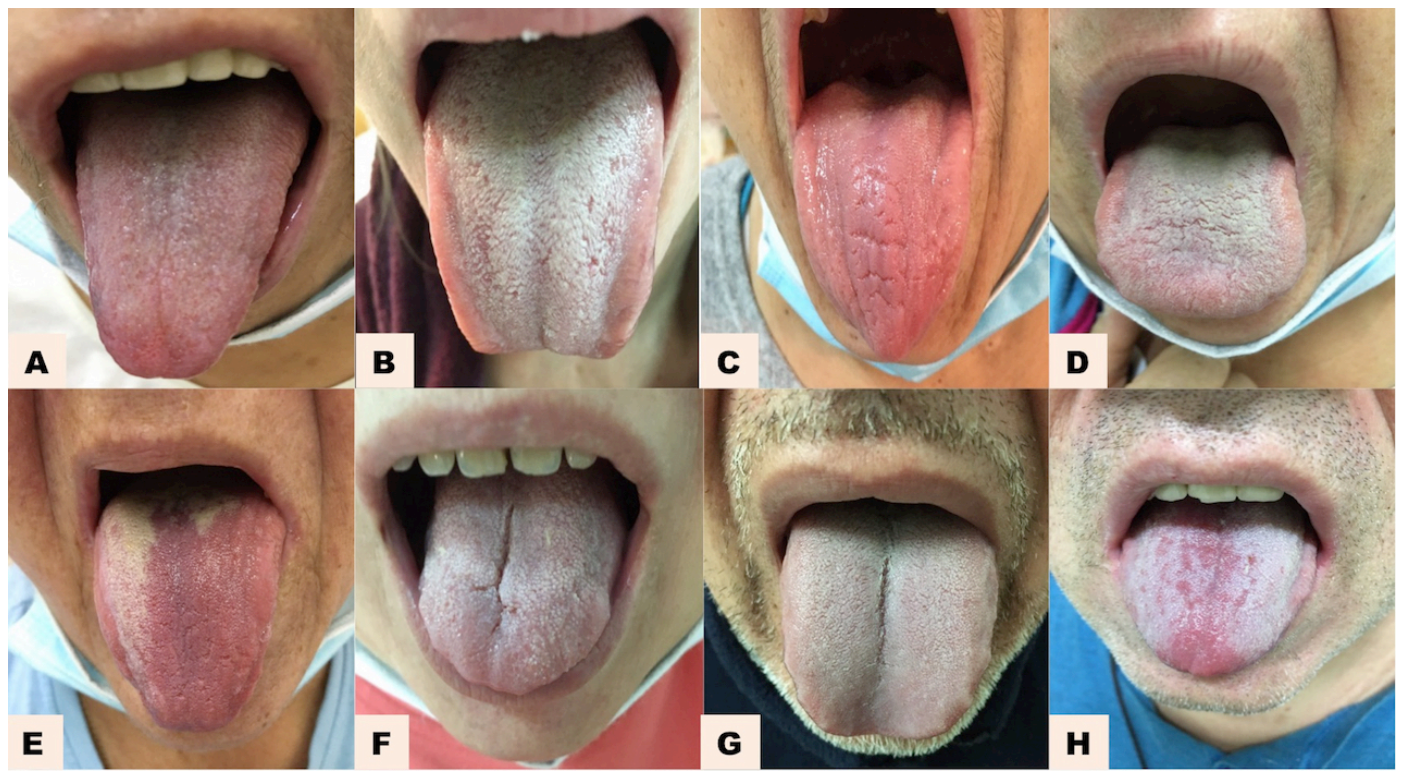

Figure 2. (A) Patient with mild severity of coronavirus disease: red tongue with no plaque; (B) Patient with mild severity of coronavirus disease: pale pink tongue with white plaque; (C) Patient with moderate severity of coronavirus disease: red tongue with no plaque; (D) Patient with moderate severity of coronavirus disease: pink tongue with grey plaque; (E) Patient with moderate severity of coronavirus disease: red tongue with yellow plaque; (F) Patient with severe coronavirus disease: pink tongue with white plaque; $(G)$ Patient with severe coronavirus disease: pink tongue with white plaque; (H) Patient with severe coronavirus disease: red tongue with white plaque. 
Statistically significant differences were observed regarding tongue color among groups of patients with different coronavirus disease severity $(\mathrm{p}<0.05)$, while an analogical trend was also noted considering tongue plaque color between mild and severe forms $(\mathrm{p}<0.05)$ and between moderate and mild forms $(\mathrm{p}<0.05)$. Patients within age groups of 41-50 years, 51-60 years, 61-70 years, 71-80 years and more than 80 years always demonstrated the presence of tongue plaque and more prevalent red and dark red color of the tongue, supreme prevalence of which was statistically significant $(\mathrm{p}<0.05)$. A similar trend was also noted regarding grey and yellow tongue plaque among the above-mentioned age groups $(\mathrm{p}<0.05)$. No gender-associated differences were found regarding changes of tongue color and tongue plaque color within analyzed study sample $(\mathrm{p}>0.05)$

\section{Discussion}

All the signs, which potentially could be classified as COVID-distinctive, should be carefully verified regarding their specificity and sensitivity as diagnostic markers, with further in-depth analysis of such at different biological organizational levels.

Considering oral mucous changes during COVID-19, some clinical cases demonstrated the development of oral ulceration and blisters formation, even though such also could be identified during other virus diseases $[17,19,21,22]$. Moreover, it should be noted that the immunological status of patients with a pronounced viral infection commonly is compromised, which also could play a role in the secondary development of oral lesions associated with underlying pathology [22]. In the brief literature review, it was found that the most frequently affected zones of the oral cavity during coronavirus disease include areas of hard palate, dorsum of the tongue and labial mucosa [17], while specifically, ulcers were the most commonly noted at the dorsum of the tongue.

Several researches demonstrated tropism relation of SARS-COV2 to the tongue and salivary glands due to the expression of angiotensin-converting enzyme 2 by their epithelial cell [34,35]. In 8 described cases of COVID-19 infection, Brandão et al. [34] collected next tongue-related manifestations: clinical case 1 painful ulcerations and necrotic ulceration of anterior dorsal tongue surface; clinical case $2-$ shallow necrotic lesions of anterior dorsal tongue part; clinical case 3 - painful ulcer lesion projected at the right margin of the tongue; clinical case $4-$ no specific tongue changes were described; clinical case 5 - multiple ulceration elements located at the apex and lateral border of tongue; clinical case 6 - no specific tongue changes were described; clinical case 7 - ulcer located at ventral part of tongue; clinical case 8 - aphthous-like ulceration with necrotic crust localized above it projected at lateral tongue surface. Provided research highlights the potential role of tongue and salivary glands as target organs regarding coronavirus invasion [34].

The biggest study related to the tongue's manifestations among COVID-patients was provided by Pang et al. [26] and included 1043 persons with different levels of coronavirus disease severity. Authors have found that tongue color changes from pale to purple were related to disease progression, analogically to tongue coating color from white to yellow and grayish-black [26]. Relations between such tongue features and coronavirus disease severity were statistically significant $(p<0.001)[26]$. An analogical trend was also noted in our study since most patients with mild disease had pale pink tongue color, while patients with severe COVID-19 infection demonstrated red and burgundy tongue color. But considering tongue plaque color, we have found yellow and gray plaque mostly presented among patients with moderate disease, while yellow and white were mostly registered among severe pathology cases. In Pang et al. [26] study, 54.90\% of patients with a moderate form of the disease were characterized with a white coating and $44.80 \%$ with yellow coating, while 
$44.90 \%$ with severe pathology were characterized with white coating, 53.74\% with yellow coating and $1.36 \%$ with grayish-black coating. So main difference between our and Pang et al. study [26] was related due to the distribution of tongue plaque colors. Such discrepancies in results could be augmented by the fact that in our study, we have not used any classification system of tongue features for manifestations differentiation, but in Pang et al. study [26], researchers used previously developed table included in Diagnostics of Chinese Medicine and Color Atlas of Chinese Medical Tongue Diagnosis. Such an approach in Pang et al. study was highly reasoned since images of the tongue further could be analyzed in a specifically designed Tongue Features Objective Research System (TFORS) [26], while in our study, we wanted to exclude the effect of interpretation error and identify the condition of each analyzed tongue on the image by fact without referring it to any classification or categorization systems. Analogically to Pang et al. study [26], we have found that patients of older age (above 40 years) are characterized with more prevalent red and dark red tongue color, present tongue plaque and yellow coating of such. Also, the amount of identified tongue plaque among COVID-positive patients was found to demonstrate greater visual accumulation in patients older than 40 years and among those with severe pathology.

Dos Santos et al. [19] presented results of ongoing COVID patient's monitoring supported by the detailed analysis of tongue changes: 1) after four weeks of hospitalization patient developed white plaque on tongue dorsum (treated with the antifungal drug but with no regression effect) with multiple pinpoint yellowish ulcers (potentially herpetic lesion); 2) two weeks after that patient was observed with a developed geographic tongue of severe manifestation form, but with no pain symptoms (areas of atrophy with adjacent yellow-white lesions elevated above the surface level), white plaque covering was fully resolved; 3) geographic tongue of moderate form was noted 10 days after discharge (44 days after hospitalization).

Such an approach of ongoing in-detail analysis of intraoral COVID-related signs used by Dos Santos et al. [19] provides a variety of perspectives for further research since such methodology seems reasonable for categorization and comparative analysis of tongue changes during coronavirus disease onset, progression and resolution. In our study, we provided just analysis of correspondence between registered COVID-19 severity and tongue color-associated manifestations, but our complex research is ongoing and at the nearest publication, it will be possible to provide results for the above-mentioned comparative analysis of COVIDassociated oral features at different stages of pathology progression.

Within the case series study structured as a Web-based questionnaire among 140 COVID-patients, just 9 persons demonstrated plaque-like changes of tongue and 4 reported swelling of the tongue [36]. Also, the authors reported a statistical correlation between plaque-like changes of the tongue and altered tongue sensation $(\mathrm{p}<0.001)$ [36]. Based on the described design of the study, all patients included in the Biadsee et al. [36] research were characterized with mild symptoms of coronavirus diseases (categorized by WHO joint report), while in our study patients demonstrated mild, moderate and severe clinical symptoms of COVID-19, associated with corresponding facts of white or no plaque present on the tongue during mild severity of pathology.

In three clinical cases described by Díaz Rodríguez et al. [37], two patients demonstrated following tongue changes which potentially could be associated with COVID-19 infection, general state of immunosuppression or influence of adjacent factors (such as stress or self-related habits): bilateral tongue surface atrophy accompanied with lateral dorsum depapillation, and tongue surface atrophy associated with fungal patch-like lesions (pseudomembranous candidiasis) and fissures localized at tongue dorsum. In addition, several studies also demonstrated the following tongue manifestation during COVID-19: small painful 
ulcerations with non-regular borders on the reddish background of anterior tongue, asymptomatic ulcers at the dorsum of the tongue, multiple macules of various size, aphthae on the ventral tongue side, tongue papilla inflammation [17,38], asymptomatic purple bulla [39].

Riad et al. [40] presented an analysis of 26 COVID-positive patients with no adjacent somathopathologies and mild or absent respiratory symptoms in which they noted the occurrence of ulcers on the dorsum, side and ventral parts of the tongue with different onset time. Tongue ulcers appeared mostly 5 days after PCR-testing (among 53.8\% patients), with 1-7 lesions per patient varying by 1-5 $\mathrm{mm}$ in size [40]. Researches also noted an interesting finding, that the localization of ulcers on the tongue was the only one in the oral cavity with such lesions absent on other oral mucous regions [40]. Irregular tongue ulcer also was mentioned as being an inaugural sign among COVID-19 patients [18].

Asymptomatic COVID-19 case presented by Corchuelo and Ulloa [41] was characterized with white zones on the posterior tongue portion and bacterial plaque accumulation at the middle third of the tongue, suggesting candidiasis diagnosis, which resolved two weeks after supportive treatment by antifungal drugs and oral hygiene measures.

In da Silva Pedrosa et al. [22] review, the authors also mentioned that xerostomia associated with reduced salivary flow among COVID-patients was noted to become more prominent with the age. In our study, we could not relate potentially present age-associated lower salivary flow with greater deposition of tongue plaque because of sanitary restrictions regarding the possibility of providing structured clinical investigation among COVID-positive patients, but such dependencies could be logically prognosticated. Also, older patients could be characterized by the possible presence of adjacent internal comorbidities, which could impact the tongue condition independently of the presence or absence of coronavirus disease.

Nevertheless, the diagnostic role of tongue during COVID-19 infection remains under debate. Resected specimen of ulcerated squamous cell lesion of the tongue of a patient with coronavirus disease demonstrated the presence of SARS-CoV-2 strain's RNA [42]. On the other hand, tongue swabs were characterized with relatively lower sensitivity for detecting SARS-CoV-2 than swabs obtained from nose or mid-turbinate [43].

For safety reasons, many physicians refrain from examining the oral cavity of patients with confirmed COVID-19. Most patients do not remove the mask from the face at all while being in the hospital. Patients themselves may simply ignore the changes of the tongue on the background of many other manifestations of COVID-19 in the acute form. Photodocumentation of tongue condition represents argumented approach for its further analysis regarding color of the tongue and color of the tongue plaque. Due to the quarantine restrictions, photoregistration of the tongue could be provided either by the patient himself or by the staff directly taking part in the treatment management of COVID-patients, with possible further transfer of such images to the dental specialist considering all the ethical aspects and issues related to personal data safety.

Ethical aspects of personal medical data use (including personal data safety issues) are reasoning the need for developing specific patient informed consent form under the condition of COVID-19 pandemic due to which doctors could investigate anonymized and blinded patients' information for the acceleration of diagnostics and treatment, provision of rapid response in life-threatening cases and relevant upgrowth of the evidence base.

Limitations of the present study related to the sanitary restrictions implemented to reduce COVID-19 spread within hospital conditions, which also influenced the possibility of more detailed analysis of other tongue-related changes, including the thickness of present plaque, tenderness of the tongue, and its' condition 
during palpation. Nevertheless, we have found that even visually identified changes of the tongue color and tongue plaque color could support coronavirus disease severity differentiation when combined with other clinical symptoms. Another limitation of present research related to the study's design, since we have evaluated tongue-associated features only once for each patient at the specific severity of coronavirus disease relevant for some certain moment. But in future studies, we will provide results of ongoing monitoring of tongue manifestations during long period screening, which will help us to verify how the color of the tongue and color of the tongue plaque are changing during pathology onset, progression and further resolution. Also, we have obtained information about coronavirus disease severity of each patient included in the study sample from the infectious disease specialists via digital information transferring to reduce the risk of infection for dentists, but in the future, it would be beneficial to analyze medical documentation of such patients with possibility to find some potential relationships not only among tongue manifestations and severity of pathology, but also among tongue signs and some other COVID-related clinical and laboratorial parameters changes.

\section{Conclusion}

Considering limitations of provided study and restrictions related to the lack of dental screening methodology developed specifically for COVID-positive patients, it could be concluded that SARS-COV-2 infection is not manifested by tongue-targeted or tongue-specific signs and features; however, coronavirus disease itself provoke changes within the tongue color and tongue plaque color similar to those registered during other internal pathologies. Taste disturbances remain the most diagnostically-prominent tonguerelated symptom during COVID-infection, nevertheless conclusion about the absence of other high-avid tongue signs during coronavirus disease should be made with caution, since the corresponding database continues to grow and some pathology-specific manifestations could be recognized at system-, organ- or tissuelevels among greater study cohort or during further epidemiological studies. Due to the received data, we may hypothesize that tongue color and color parameters of tongue plaque related to the COVID-infection severity with positive type of interrelation: red tongue color and prominent white-yellow tongue plaque were identified the most frequently among severe disease cases. However, further clinical investigations should be provided to distinguish tongue-related manifestations on such, caused specifically by COVID-infection, and such, associated with the effect of provided treatment.

\section{Authors' Contributions}

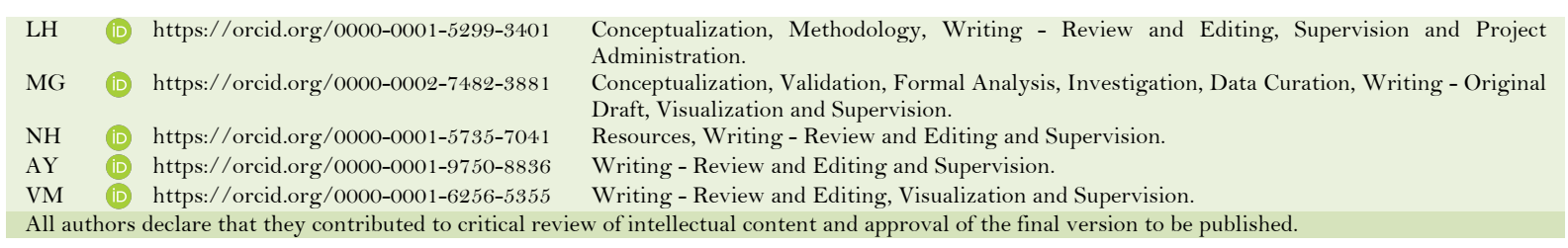

\section{Financial Support}

None.

\section{Conflict of Interest}

The authors declare no conflicts of interest.

\section{Data Availability}

The data used to support the findings of this study can be made available upon request to the corresponding author. 


\section{References}

[1] Seerangaiyan K, Jüch F, Winkel EG. Tongue coating: its characteristics and role in intra-oral halitosis and general health - a review. J Breath Res 2018; 12(3):034001. https://doi.org/10.1088/1752-7163/aaa3a1

[2] Wang X, Zhang B, Yang Z, Wang H, Zhang D. Statistical analysis of tongue images for feature extraction and diagnostics. IEEE Trans Image Process 2013; 22(12):5336-47. https://doi.org/10.1 109/TIP.2013.2284070

[3] Jung CJ, Jeon YJ, Kim JY, Kim KH. Review on the current trends in tongue diagnosis systems. Integr Med Res 2012; 1(1):13-20. https://doi.org/10.1016/j.imr.2012.09.001

[4] Anastasi JK, Chang M, Quinn J, Capili B. Tongue inspection in TCM: observations in a study sample of patients living with HIV. Med Acupunct 2014; 26(1):15-22. https://doi.org/10.1089/acu.2013.1011

[5] Zhang B, Kumar BV, Zhang D. Detecting diabetes mellitus and nonproliferative diabetic retinopathy using tongue color, texture, and geometry features. IEEE Trans Biomed Eng 2013; 61(2):491-501. https://doi.org/10.1109/TBME.2013.2282625

[6] Gaddey HL. Oral manifestations of systemic disease. Gen Dent 2017; 65(6):23-9.

[7] Zhang B, Wang X, You J, Zhang D. Tongue color analysis for medical application. Evid-Based Complement Alternat Med 2013; 2013:1-11. https://doi.org/10.1155/2013/264742

[8] Kawanabe T, Kamarudin ND, Ooi CY, Kobayashi F, Mi X, Sekine M, et al. Quantification of tongue colour using machine learning in Kampo medicine. Eur J Integr Med 2016; 8(6):932-41. https://doi.org/10.1016/j.eujim.2016.04.002

[9] Velasco J, Rojas J, Ramos JP, Muaña HM, Salazar KL. Health evaluation device using tongue analysis based on sequential image analysis. IJATCSE 2019; 8(3):451-7. https://doi.org/10.30534/ijatcse/2019/19832019

[10] Wang ZC, Zhang SP, Yuen PC, Chan KW, Chan YY, Cheung CH, et al. Intra-rater and inter-rater reliability of tongue coating diagnosis in traditional chinese medicine using smartphones: Quasi-delphi study. JMU 2020; 8(7):e16018. https://doi.org/10.2196/16018

[11] Tania MH, Lwin K, Hossain MA. Advances in automated tongue diagnosis techniques. Integr Med Res 2019; 8(1):4256. https://doi.org/10.1016/j.imr.2018.03.001

[12] Kim SY, Byun JS, Choi JK, Jung JK. A case report of a tongue ulcer presented as the first sign of occult tuberculosis. BMC Oral Health 2019; 19(1):1-5. https://doi.org/10.1186/s12903-019-0764-y

[13] Jain P, Jain I. Oral manifestations of tuberculosis: step towards early diagnosis. J Clin Diag Res 2014; 8(12):ZE18. https://doi.org/10.7860/JCDR/2014/10080.5281

[14] Souza PV, Pinto WB, Oliveira AS. Bright tongue sign: a diagnostic marker for amyotrophic lateral sclerosis. Arq Neuro-Psiquiatr 2014; 72(7):572. https://doi.org/10.1590/0004-282X20140077

[15] Ayinampudi BK, Gannepalli A, Pacha VB, Kumar JV, Khaled S, Naveed MA. Association between oral manifestations and inhaler use in asthmatic and chronic obstructive pulmonary disease patients. J Dr NTR Univ Health Sci 2016; $5(1): 17$. https://doi.org/10.4103/2277-8632.178950

[16] World Health Organization. Coronavirus Disease (COVID-19) Dashboard. 2021. Available from: https://covid 19.who.int/. [Accessed on January 13, 2020].

[17] Halboub E, Al-Maweri SA, Alanazi RH, Qaid NM, Abdulrab S. Orofacial manifestations of COVID-19: a brief review of the published literature. Braz Oral Res 2020; 34:e124. https://doi.org/10.1590/1807-3 107bor-2020.vol34.0124

[18] Chaux-Bodard AG, Deneuve S, Desoutter A. Oral manifestation of Covid-19 as an inaugural symptom?. J Oral Med Oral Surg 2020; 26(2):18. https://doi.org/10.1051/mbcb/2020011

[19] dos Santos JA, Normando AG, da Silva RL, De Paula RM, Cembranel AC, Santos-Silva AR, et al. Oral mucosal lesions in a COVID-19 patient: new signs or secondary manifestations?. Int J Infect Dis 2020; 97:326-8. https://doi.org/10.1016/j.ijid.2020.06.012

[20] Horzov L, Goncharuk-Khomyn M, Kostenko Y, Melnyk V. Dental Patient Management in the Context of the COVID-19 Pandemic: Current Literature Mini-Review. Open Public Health J 2020; 13(1):459-63. https://doi.org/10.2174/1874944502013010459

[21] da Silva Pedrosa M, Sipert CR, Nogueira FN. Altered taste in patients with COVID-19: the potential role of salivary glands. Oral Dis 2020; Suppl 3:798-800. https://doi.org/10.1111/odi.13496

[22] da Silva Pedrosa M, Sipert CR, Nogueira FN. Salivary glands, saliva and oral findings in COVID-19 infection. Pesqui Bras Odontopediatria Clín Integr 2020; 20:e0 104. https://doi.org/10.1590/pboci.2020.112

[23] Yan CH, Faraji F, Prajapati DP, Boone CE, De Conde AS. Association of chemosensory dysfunction and Covid-19 in patients presenting with influenza-like symptoms. Int Forum Allergy Rhinol 2020; 10(7):806-13. https://doi.org/10.1002/alr.22579

[24] Wang B, Pang K, Chen S, Gong J, Deng J, Liu J. A preliminary study of tongue image in 78 patients with COVID-19. Jiangsu Tradit Chin Med 2020; 53:84-6. https://doi.org/10.19844/j.cnki.1672-397X.2020.04.014

[25] Zhou G, Huang D, Cai Y, Huang K, Xie D. Relationship between tongue characteristics and clinical typing in COVID-19 patients. J Tradit Chin Med 2020:1-4.

[26] Pang W, Zhang D, Zhang J, Li N, Zheng W, Wang H, et al. Tongue features of patients with coronavirus disease 2019: a retrospective cross-sectional study. Integr Med Res 2020; 9(3):100493. https://doi.org/10.1016/j.imr.2020.100493 
[27] World Health Organization. Clinical management of COVID-19 (interim guidance). 2021. Available from: https://www.who.int/publications/i/item/clinical-management-of-covid-19. [Accessed on January 13, 2020].

[28] Tatem KS. Comparing prevalence estimates from population-based surveys to inform surveillance using electronic health records. Prev Chronic Dis 2017; 14:E44. https://doi.org/10.5888/pcd14.160516

[29] van Baal PH, Engelfriet PM, Hoogenveen RT, Poos MJ, van den Dungen C, Boshuizen HC. Estimating and comparing incidence and prevalence of chronic diseases by combining GP registry data: the role of uncertainty. BMC Public Health 2011; 11(1):163. https://doi.org/10.1186/1471-2458-11-163

[30] Tekindal MA, Gullu O, Yazici AC, Yavuz Y. The Cochran-Armitage test to estimate the sample size for trend of proportions for biological data. Turk J Field Crops 2016; 21(2):286-97. https://doi.org/10.17557/tjfc.33765

[31] Buonaccorsi JP, Laake P, Veierød MB. On the power of the Cochran-Armitage test for trend in the presence of misclassification. Stat Methods Med Res 2014; 23(3):218-43. https://doi.org/10.1177/0962280211406424

[32] McHugh ML. Interrater reliability: the Kappa statistic. Biochem Med 2012; 22(3):276-82.

[33] Hsu LM, Field R. Interrater agreement measures: Comments on Kappan, Cohen's Kappa, Scott's $\pi$, and Aickin's $\alpha$. Understanding Statistics 2003; 2(3):205-19. https://doi.org/10.1207/S15328031US0203_03

[34] Brandão TB, Gueiros LA, Melo TS, Prado-Ribeiro AC, Nesrallah AC, Prado GV, et al. Oral lesions in patients with SARS-CoV-2 infection: could the oral cavity be a target organ?. Oral Surg Oral Med Oral Pathol Oral Radiol Endod 2020; S22 12-4403(20)31119-6. https://doi.org/10.1016/j.00oo.2020.07.014

[35] Nemeth-Kohanszky ME, Matus-Abásolo CP, Carrasco-Soto RR. Manifestaciones orales de la infección por COVID19. Int J Odontostomat 2020; 14(4):555-60. https://doi.org/10.4067/So718-381X2020000400555

[36] Biadsee A, Biadsee A, Kassem F, Dagan O, Masarwa S, Ormianer Z. Olfactory and oral manifestations of COVID-19: sex-related symptoms - a potential pathway to early diagnosis. Otolaryngol Head Neck Surg 2020; 163(4):722-8. https://doi.org/10.1177/0194599820934380

[37] Rodríguez MD, Romera AJ, Villarroel M. Oral manifestations associated with COVID-19. Oral Dis 2020 ; 1-3. https://doi.org/10.1111/odi.13555

[38] Liang K, Huang X, Chen H, Qiu L, Zhuang Y, Zou C, et al. Tongue diagnosis and treatment in traditional Chinese medicine for severe COVID-19: a case report. Ann Palliat Med 2020; 9(4):2400-7. https://doi.org/10.2 1037/apm-20-1330

[39] Cruz Tapia RO, Peraza Labrador AJ, Guimaraes DM, Matos Valdez LH. Oral mucosal lesions in patients with SARSCoV-2 infection. Report of four cases. Are they a true sign of COVID-19 disease?. Spec Care Dent 2020; 40(6):555-60. https://doi.org/10.1111/scd.12520

[40] Riad A, Klugar M, Krsek M. COVID-19-related oral manifestations: early disease features?. Oral Dis 2020. https://doi.org/10.1111/odi.13516

[41] Corchuelo J, Ulloa FC. Oral manifestations in a patient with a history of asymptomatic COVID-19: Case report. Int J Infect Dis 2020; 100:154-7. https://doi.org/10.1016/j.ijid.2020.08.071

[42] Guerini-Rocco E, Taormina SV, Vacirca D, Ranghiero A, Rappa A, Fumagalli C, et al. SARS-CoV-2 detection in formalin-fixed paraffin-embedded tissue specimens from surgical resection of tongue squamous cell carcinoma. J Clin Pathol 2020; 73(11):754-7. https://doi.org/10.1136/jclinpath-2020-206635

[43] Tu YP, Jennings R, Hart B, Cangelosi GA, Wood RC, Wehber K, et al. Swabs collected by patients or health care workers for SARS-CoV-2 testing. N Engl J Med 2020; 383(5):494-6. https://doi.org/10.1056/NEJMc201632 1 